\title{
Wireless Information and Power Transfer in Multiuser OFDM based Systems
}

\author{
Sakshi Sharma \\ Research Scholar, Mahakal Institute of \\ Technology, Ujjain (M.P.)
}

\author{
Gaurav Gupta \\ Mahakal Institute of Technology, \\ Ujjain (M.P.)
}

\begin{abstract}
In this paper, we have study the optimal design for synchronous wireless information and power transfer (SWIPT) in downlink multiuser orthogonal frequency division multiplexing (OFDM) systems, where the users gather energy and explain information by using the same type of signals received from a stable access point (AP). For the transmission of information, we have to consider two types of numerous access patterns, called, time division multiple access (TDMA) and orthogonal frequency division multiple access (OFDMA). Beneath the above two scenarios, we locate the problem of maximizing the weighted sum-rate overall users by varying the time or frequency power arrangement and either TS or PS ratio, subjected to a minimum harvested energy constraint on each user as well as a highest and or overall transmission power constraint.. It is revealed that the highest power constraint inflict on each OFDM SC as well as the number of users in the system play basic roles in the rateenergy performance comparison by the two types of proposed arrangements.
\end{abstract}

\section{Keywords}

OFDM, Sub- Carrier allocation, TDMA, Power splitting, time Switching.

\section{INTRODUCTION}

In order to fulfill the requirement of the exponential growing demand of wireless multimedia services, a high or maximum speed data access is a necessity condition. Hence, various types of techniques have been proposed in present years to achieve maximum system capacities. Among of them, we are interested to the multiple-input multiple- output that we called MIMO in short. The MIMO concept has attracted lot of variations in the wireless communications due to its potential to maximize the system capacity rate without any extra bandwidth. Multipath proliferation generally causes selective frequency channels. To compare the effect of frequency assortment fading, MIMO is associated with orthogonal frequency-division multiplexing in short OFDM, method. OFDM is a modulation procedure which converts frequency into assortment channel into a set of corresponding flat fading channels. A cyclic prefix CP is added at the starting of each OFDM symbol to removed ICI and ISI. The inserted cyclic prefix is longer than equal to the channel.

\subsection{OFDM}

Orthogonal Frequency Division Multiplex, under this the modulation benchmark is being used for various wireless and radio communications such as radio applications from DAB, DVB, Wi-Fi and also in Mobile Video. Orthogonal Frequency Division Multiplex i.e. OFDM is a modulation format that is finding increasing levels of use in today's radio communications scenario. OFDM has been access in the Wi$\mathrm{Fi}$ group where the 802.11 is an important and standard uses .It is also to be provided data rates up to $54 \mathrm{Mbps}$ in the 5
GHz ISM is also termed as Industrial, Scientific and Medical band. In addition to this the recently ratified $802.11 \mathrm{~g}$ benchmark has it in the $2.4 \mathrm{GHz}$ ISM band. If this thing was not sufficient, it is also being utilized for digital earthbound television transmissions as well as DAB digital radio. A advanced arrangement of broadcasting called Digital Radio Mondi ale for the long,medium and short wave bands is being to be discovered as well as launched and this has also access COFDM. Then for the further process it is being to be proposed as the modulation arrangementfor fourth generation cell phone systems that are in their starting stages of the development .An OFDM is also being used for several of the proposed mobile phone video systems. OFDM, orthogonal frequency division multiplex is a preferably different pattern for modulation to that used for more traditional forms of transmission. It can already utilizes various carriers together to provide several advantages over than the easier modulation scheme.

An OFDM signal consists a large number of closely spaced modulated carriers. When modulation of any form - voice, data, etc. is acquire to a carrier, and then sidebands spread out on the either side of modulation. It is a compulsory condition for a receiver to be capable to found the entire signal that provides us successfully demodulates the data. Due to which, when signals are to be transmitted close to one another it should be check that they must be provided gap so that the receiver can separate them by using a filter and in between them there must be a guard band. This is not the situation of an OFDM. Although the sidebands from every carrier overlap, they can be constantly obtained without any of the interference that might be expected because they are orthogonal to each another. This can be obtained by having the carrier spacing that will be equal to the reciprocal of the symbol period.

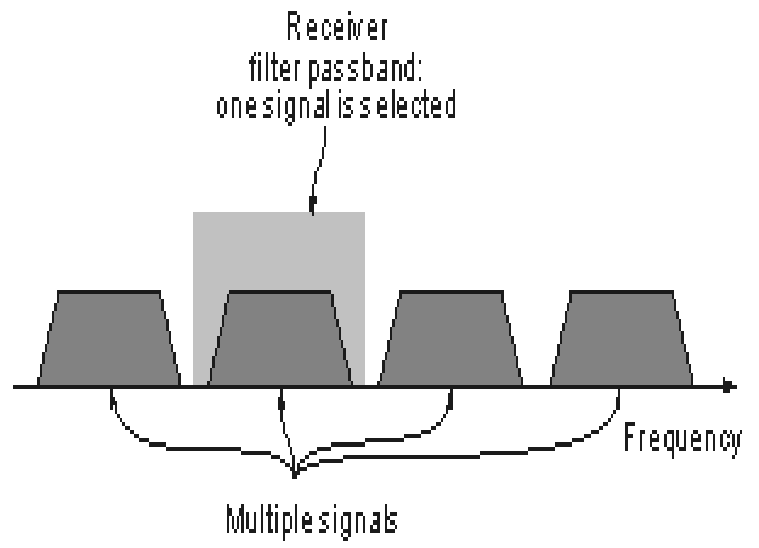

Fig.1.1.Traditional view of receiving signals carrying modulation 
To visualize how OFDM can operate or works, it is compulsory to look at the receiver. This can acts as a embankment of demodulators, transcribing each carrier down to DC. The obtained signal is integrated over the symbol period to reanimate the data from that carrier. The same demodulator can demodulates the other carriers also. As the carrier gaping equal to the reciprocal of the symbol period means that they will have a whole number of cycles in the symbol period and their involvement will sum to zero or we can say in other words, there is no interference of contribution.

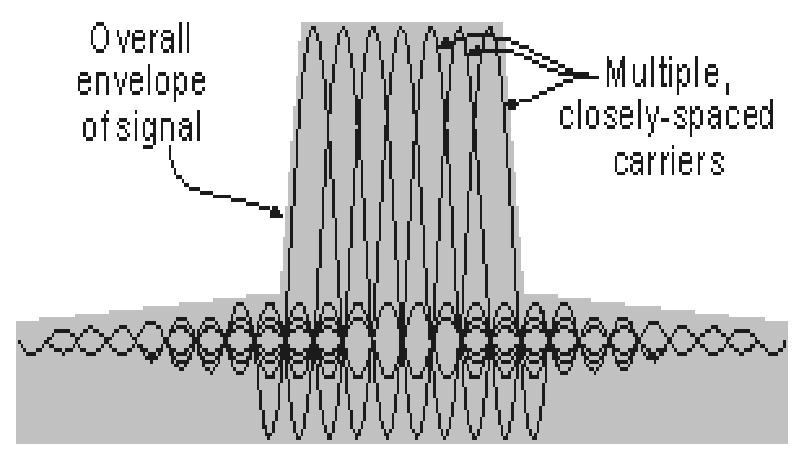

Fig.1.2.OFDM Spectrum

One of the basic requirements of the OFDM receiving and transmitting systems is that they should be linear. In terms of the equipment that is to be used the maximum peak to the average ratio of the multi-carrier systems such as OFDM acquires the RF terminal amplifier on the output of the transmitter to be capable to handle the peaks while the average power is much less and this will leads to inefficiency. In few systems the peaks are restricted. Although this established distortion that system can rely on the error correction to eliminate them.

\subsection{Data on OFDM}

The data is to be transmitted on an OFDM signal is distributed among the carriers of the signal, each carrier taking portion of the payload. Due to which it eliminates the data rate taken by each carrier. The lower rate of data has an effective and efficient advantage and its interference from reflections is much less critical. This can be obtained by the addition of guard band time period or guard interval into the system. This yields that the data is analyzed only when the signal is in stable condition and even no new form of delayed signals are arrived that would recognize the timing and phase of the signal.

The OFDM transmission pattern has the following basic advantages:

1. Make efficient use of the spectrum by allowing overlapping.

2. By designating the channel into a limited band flat fading sub channels, OFDM is more defiance to frequency selective fading than single carrier systems are.

a. Eliminates ISI and IFI through use of a cyclic prefix.

b. Using adequate channel coding and interleaving one can recover symbols lost due to the frequency selectivity of the channel. c. Channel equalization becomes simpler than by using adaptive equalization techniques with single carrier systems.

d. It is possible to use largest likelihood understand with reasonable complexity, as discussed in OFDM is computationally efficient by using FFT method tocontraption the modulation and demodulation functions.

e. In conjunction with differential modulation ,there is no requirement to implement a channel estimator.

f. Is it less sensitive to sample timing offsets than single carrier systems are.

g. Provides good protection against co-channel interference and continuous parasitic noise.

\section{METHODS USED}

1. Guard Interval-OFDM variants numerous path effects or interference on a given frequency only affects a small number of the carriers, the remaining ones are being obtained correctly. By the use of error-coding methods, in which addition of further format of data to the transmitted signal. It enables several or all of the corrupted data to be redesigned within the receiver. This can be completed because of the error correction code is to be transmitted in a various portion of the signal.

2. VOFDM: Vector OFDM. This format of OFDM uses the concept of MIMO i.e. Multiple input Multiple output technology. It is being generated by CISCO Systems. It can uses numerous forms of antennas to transmit and receive the signals for example multi-path effects that can be utilized to increase the signal reception and also improve the transmission speeds that can be supported efficiently.

3. WOFDM: Wideband OFDM. The abstraction of this form of OFDM is such that it uses a degree of gaping between the channels that is large enough to that of any frequency errors in between the transmitter and receiver, do not affect the performance level. It is specifically applicable to Wi-Fi systems. Every format of OFDM utilizes the previous or same basic notion of using close spaced orthogonal carriers and each have carrying low form of data rate signals. During the demodulation phase the data is then combined to provide the complete signal.

\section{SIMULATION RESULT}

OFDM is universally used in conjunction with channel coding, it is also termed as 'correction 'and always uses almost frequency and or time interleaving. Frequency or subcarrier intersperse increases resistance to frequency-selective channel, cases such as fading. Like, when a portion of the channel bandwidth fades, frequency interleaving provides that the bit errors that would give result from those subcarriers in the faded portion of the bandwidth are distributed elsewhere in the bit-stream rather than being in the concentrated manner. Similarly, time interleaving provides that bits that are really close together in the bit-stream are transmitted distance apart in time. Thus mitigating against extreme fading as would happen when travelling at high speed. However, time interleaving is of less benefit in comparison to slowly fading channels, for example for stationary reception, and frequency interleaving provides less to no benefit for narrowband channels that agonize from flat-fading (where the overall channel bandwidth fades at the same time). 
The reason why interleaving is used on OFDM is to attempt to distribute the errors outside in the bit-stream that is dispense in the error correction decoder, because when such type of decoders are presented with a high concentration of mistakes the decoder is not capable to correct entire bit errors, and a burst of uncorrected mistakes can takes place. A same type of design of audio data encoding creates compact disc (CD) playback with resistance.

A traditional kind of error correction coding used in addition with the OFDM-depended systems is in the form of convolution coding, often concatenated with Reed-Solomon coding. Generally, additional interleaving that is on top of the time and frequency interleaving mentioned above, is in between the two layers of coding can be implemented. The option for Reed-Solomon coding as the external error correction code is depend on the observation that the Viterbi decoder used for inner convolution decoding gives short form of errors bursts when there is a high concentration of errors are to be obtained, and Reed-Solomon codes are intrinsically well adaptive to correcting bursts of errors.

\section{CONCLUSION}

This paper has contain complete study of the resource arrange optimization for a multiuser OFDM-based downlink SWIPT system. There are basically two transmission schemes are to be investigated, namely, the TDMA depended information transmission with TS should be applied at each receiver, and the information based on transmission with PS applied at each receiver. Our study supports that, for the TS scheme, the system can be achieved the same rate as the traditional TDMA system, and at the same time every user is still able to yield a reasonable value of energy. When the yielded form of energy required at users is sufficiently high, however, a nonzero EH slot may be needed. This in turn reduces the rate of the TS scheme significantly. Hence, the PS scheme may outperform the TS scheme when the gathered energy is sufficiently large. Furthermore, in practical circuits the power splitter or switcher may introduce insertion loss and demean the performance of the two patterns. This issue is not located in this paper, and is left for later on work.

\section{REFERENCES}

[1] A. J. Paulraj, D. A. Gore, R. U. Nabar, and H. Bolcskei, "An overview ofMIMO communications - A key to gigabit wireless," Proc. IEEE, vol. 92,no. 2, pp. 198-218, Feb. 2004.

[2] B. Muquet, Z. Wang, G. B. Giannakis, M. de Courville, andP. Duhamel, "Cyclic prefixing or zero padding for wireless multicarrier transmissions?" IEEE Trans. Commun., vol. 50, no. 12, pp. 2136-2148, Dec.2002.

[3] Y. Li, N. Seshadri, and S. Ariyavisitakul, "Channel estimation for OFDM systems with transmitter diversity in mobile wireless channels," IEEE J. Sel. Areas Commun., vol.17, no.3, pp.461-471,March 1999.

[4] D. Wan, B. Han, J. Zhao, X. Gao, and X. You, "Channel estimation algorithms for broadband MIMO-OFDM sparse channel," Proc.14th IEEE Int. Symp. on Personal, Indoor and Mobile Radio Communications, pp.19291933, Beijing, China, Sept. 2003.

[5] 3GPP, "Evolved Universal Terrestrial Radio Access (EUTRA); Physical channels and modulation," TS 36.211, 3rd Generation Partnership Project (3GPP), Sept. 2008.
[6] J.-J. van de Beek, O. Edfors, M. Sandell, S. K. Wilson, and P. O. Borjesson, "On channel estimation in OFDM systems," in Proc. IEEE45th Vehicular Technology Conf., Chicago, IL, Jul.1995, pp. 815-819.

[7] O. Edfors, M. Sandell, J.-J. van de Beek, S. K. Wilson and P. O. Borjesson, "OFDM channel estimation by singular value decomposition,"in Proc. IEEE 46th Vehicular Technology Conference, Atlanta, GA, USA,Apr. 1996, pp. 923-927.

[8] S. D. Ma and T. S. Ng, "Time domain signal detection based on second-order statistics for MIMOOFDM systems,” IEEE Trans.Signal Process. vol.55, no.3,pp.1150-1158,Mar.2007.

[9] S.D. Ma and T.S. Ng, "Semi-blind time-domain equalization for MIMO-OFDM systems", IEEE Transactions on Vehicular Technology, 57(4), 22192227, July, 2008

[10] MM Rana"Channel estimation techniques and LTE Terminal implementation challenges" in Proc. International Conference on Computer and Information Technology pp. 545-549 December 2010

[11] M. Simko, D. Wu, C. Mehlführer, J. Eilert, D. Liu "Implementation Aspects of Channel Estimationfor 3GPP LTE Terminals" in Proc. Proc. European Wireless 2011, Vienna, April, 2011

[12] C. Budianu and L. Tong, "Channel estimation for spacetime orthogonal block codes", IEEE Trans. Signal Process., Vol. 50, pp. 2515-2528, Oct, 2002.

[13] M. Biguesh and A. B. Gershman, "MIMO channel estimation: optimal training and tradeoffs between estimation techniques," Proc. ICC'04, Paris, France, June 2004.

[14] M. Biguesh and A. B. Gershman, "Training-based MIMO channel estimation: a study of estimator tradeoffs and optimal training signals," IEEE Trans. Signal Processing, Vol. 54, No. 3, Mar. 2006.

[15] H.Z.Y. Li, A. Reid and J. Terry, "Channel estimation for MIMO OFDM in correlated fading channels", Proc. ICC'05, Vol. 4, pp. 2626 - 2630, May 2005.

[16] Y. (G.) Li "Simplified channel estimation for OFDM systems with multiple transmit antennas," IEEE Trans. Wireless Communications, vol. 1, pp. 67-75, Jan. 2002

[17] I. Barhumi, G. Leus and M. Moonen, "Optimal training design for MIMO OFDM systems in mobile wireless channels," IEEE Trans. Signal Processing, vol. 51, pp. 1615-1624, June 2003

[18] W. C. Jakes, Microwave Mobile Communications, New York: John Wiley \& Sons, 1974.

[19] A. F. Naguib, "Adaptive antenna for CDMA wireless network," Ph.D.dissertation, Stanford Univ., Palo Alto, CA, Aug. 1996

[20] J. Tsai, R. M. Buehrer and B. D. Woerner, "Spatial fading correlation function of circular antenna arrays with Laplacian Energy distribution," IEEE Comms. Letters, vol. 6, No. 5, May 2002 